\title{
Identification and Physiochemical Analysis of ERK Interacting Proteins Using Bio-Computational Tools
}

\author{
Khuleshwari Kurrey, Vijay Paramanik* \\ Cellular and Molecular Neurobiology \& Drug Targeting Laboratory, Department of Zoology, \\ Indira Gandhi National Tribal University, Amarkantak, Madhya Pradesh, India \\ Email: *vijayparamanik@gmail.com
}

How to cite this paper: Kurrey, K. and Paramanik, V. (2018) Identification and Physiochemical Analysis of ERK Interacting Proteins Using Bio-Computational Tools. World Journal of Neuroscience, 8 , 303-313.

https://doi.org/10.4236/wjns.2018.82024

Received: March 28, 2017

Accepted: May 19, 2018

Published: May 22, 2018

Copyright ( 92018 by authors and Scientific Research Publishing Inc. This work is licensed under the Creative Commons Attribution International License (CC BY 4.0).

http://creativecommons.org/licenses/by/4.0/

\begin{abstract}
ERK (Extracellular Signal Regulated Kinase) or MAP kinase is an intracellular signaling molecule. ERK is involved in regulation of various functions i.e. cell proliferation, cell migrations, cell survival and many more. It gets activated in response of various stimuli like growth factors, cytokines, virus, second messengers, transforming agents and carcinogens. While transferring signals from cell surface receptors to cell nucleus, ERK interacts with a numbers of proteins. Physiochemical and functional characterization of these proteins is little known. Thus, we attempted to study physiochemical and functional properties of ERK interacting proteins using bio-computational tools. ExPASy and SOSUI server suggested 22 ERK interacting proteins. Physical and chemical parameters of these ERK interacting partners indicated higher percentage of hydrophobic amino acid and leucine as major constituent. Moreover, the instability index indicated that four proteins are stable in over wide range temperature in vitro, and remaining eighteen proteins were found unstable. In addition, SOSUI server showed that fifteen proteins were soluble and six are trans-membrane in nature.
\end{abstract}

\section{Keywords}

Extracellular Signal Regulated Kinase (ERK), GRAVY, SOSUI, STRING, STITCH, ExPASy

\section{Background}

ERK (Extracellular Signal Regulated Kinase) or MAP kinase is an intracellular signaling molecule. It is associated to regulate various cell functions, namely cell proliferation, cell migrations, cell survival and many other biological functions. 
ERK gets activated in response to the response of various stimuli such as different growth factors, cytokines, virus, second messengers, transforming agents and carcinogens. ERK helps to transfer signals from cell surface receptors to nucleus of the cells. However, it's still undefined how single ERK pathway regulates verity of specific and different cellular functions [1]. ERK interacts with wide range of proteins to perform specific function. It is important to line up the action of ERK with its interactive proteins at molecular level. Thus the physiochemical and functional properties are needed.

With increasing knowledge in bioinformatics, the computational server is used to determine physical, chemical and functional properties of proteins. These services are useful to facilitate researchers to frame numerous experiments at molecular level. Although physiochemical and functional studies of various proteins and drugs have been done by many workers using bioinformatics tools, [2] computational study on ERK interactive proteins is little studied. Thus, we focused to study physiochemical and functional characterization of ERK interactive proteins using computational tools. Such studies are helpful to understand ERK dependent signal transduction.

\section{Methodology}

The methodology was followed in this manuscript as under:

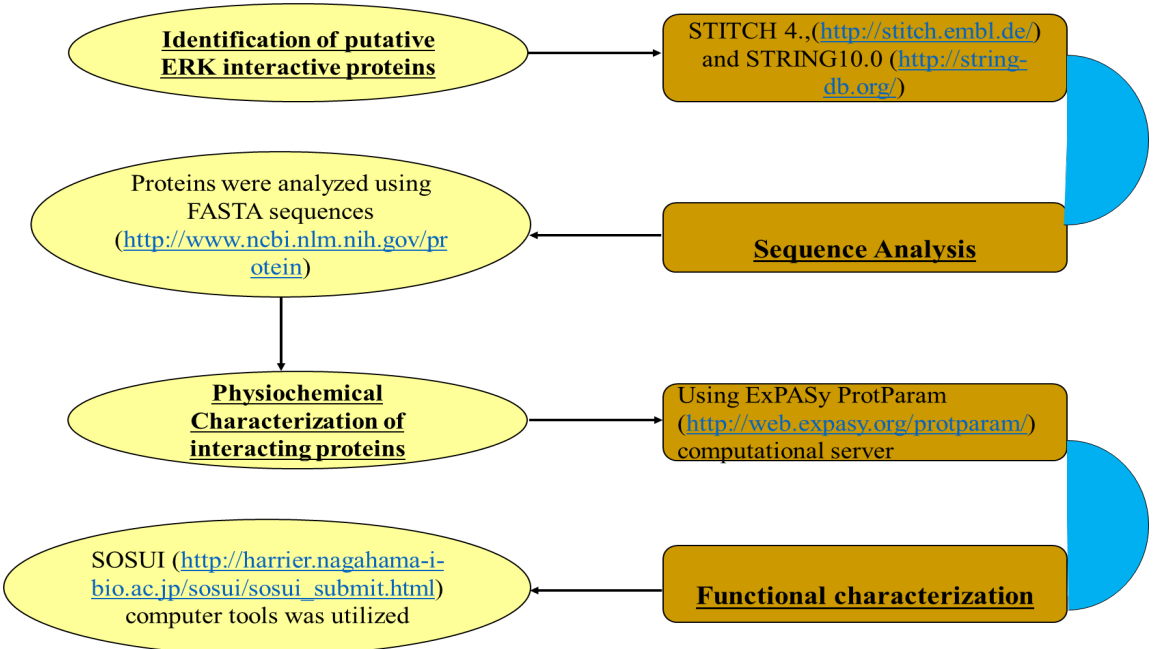

\subsection{Identification of Putative ERK Interactive Proteins}

ERK interactive proteins were retrieved by using STITCH 4., which is a hugearchives of protein-chemical interaction (http://stitch.embl.de/) [3] and STRING 10.0 (http://string-db.org/), is a huge archives of protein-protein interactions [4] [5]. These tools predict 42ERK interactive proteins. Physiochemical analysis of 22 commonly predicted proteins by both servers were done (Table 1 ).

\subsection{Sequence Analysis}

The proteins were analyzed using FASTA sequences and proteins accession 
Table 1. Interacting proteins of ERK retrieved from STITCH and STRING.

\begin{tabular}{|c|c|c|c|}
\hline S.N. & $\begin{array}{c}\text { NCBI Accession } \\
\text { no. }\end{array}$ & Gene Name & Protein Name \\
\hline 1 & NP_004433.2 & ЕРНB2 & ephrin type-B receptor 2 isoform 2 precursor \\
\hline 2 & NP_620407.1 & MAPK1 & mitogen-activated protein kinase 1 \\
\hline 3 & NP_060033.3 & IL17RD & interleukin-17 receptor $\mathrm{D}$ precursor \\
\hline 4 & NP_001093207.1 & WDR83 & WD repeat domain-containing protein 83 \\
\hline 5 & AAH15221.1 & TESC & tescalcin \\
\hline 6 & AAI36277.1 & MAP3K4 & mitogen-activated protein kinase kinasekinase 4 \\
\hline 7 & EAX07758.1 & MAP3K6 & mitogen-activated protein kinase kinasekinase 6 \\
\hline 8 & AAQ89028.1 & ULBP2 & UL16 binding protein 2 \\
\hline 9 & AAK13081.1 & ULBP1 & UL16 binding protein 1 \\
\hline 10 & CAG38739.1 & DUSP14 & dual specificity phosphatase 14 \\
\hline 11 & BAA34369.1 & DUSP6 & dual specificity phosphatase 6 \\
\hline 12 & AAI08905.1 & RHAMM & hyaluronan-mediated motility receptor \\
\hline 13 & NP_001136254.1 & MAGI3 & membrane-associated guanylate kinase \\
\hline 14 & CAG46533.1 & PEA15 & phosphoprotein enriched in astrocytes 15 \\
\hline 15 & AAH95453.1 & WARS & tryptophanyl-tRNA synthetase, cytoplasmic \\
\hline 16 & AAH60837.1 & DUSP9 & dual specificity phosphatase 9 \\
\hline 17 & NP_005912.1 & MAP3K1 & mitogen-activated protein kinase kinasekinase 1 \\
\hline 18 & AAK13083.1 & ULBP3 & UL16 binding protein 3 \\
\hline 19 & NP_067004.3 & SLAMF7 & SLAM family member 7 isoform a precursor \\
\hline 20 & NP_002410.1 & MAP3K11 & mitogen-activated protein kinase kinasekinase 11 \\
\hline 21 & NP_065789.1 & KIDINS220 & kinase $\mathrm{D}$-interacting substrate, $220 \mathrm{kDa}$ \\
\hline 22 & Q6VAB6.2 & KSR2 & kinase suppressor of ras 2 \\
\hline
\end{tabular}

number (http://www.ncbi.nlm.nih.gov/protein) [6] [7] [8] [9] from NCBI.

\subsection{Physiochemical Characterization of Interacting Proteins}

Using ExPASy ProtParam (http://web.expasy.org/protparam/) computational server, primary structure and physiochemical characters of proteins, such as amino acids number (AA), Molecular weight of protein, Theoretical pI, Amino acids compositions of protein, total number of negatively charged residues (Asp + Glu), total number of positively charged residues (Arg + Lys), extinction coefficient, instability index, aliphatic index and Grand Average Hydropathicity (GRAVY) were observed.

\subsection{Functional Characterization}

The nature of proteins whether it is soluble or trans membrane in character, SOSUI (http://harrier.nagahama-i-bio.ac.jp/sosui/sosui_submit.html) computer 
tools was utilized to analyze them [10].

\section{Results}

\subsection{Physiochemical Characterization}

ERK interactive protein's amino acids (AA) composition was presented in Table 2 and Table 3. Most of the ERK interacting proteins are hydrophilic in nature. AA composition indicates that higher percentage of hydrophobic amino acid leucine. This is due to presence of all the hydrophobic proteins inside the cell membrane and hydrophilic amino acids on the outside. Sequence length of protein represents basic nature of protein [6] [7] [8].

Theoretical $\mathrm{pI}$ (isoelectric point) (Table 4) value of 17 proteins i.e. EPHB2, MAPK1, IL17RD, WDR83, TESC, MAP3K4, MAP3K6, ULBP2, ULBP1, DUSP6, RHAMM, KIDINS220, PEA15, WARS, DUSP9, MAP3K1, SLAMF7 is less than 7. Hence, these all are acidic in nature and other 5 proteins i.e. KSR2, DUSP14, MAGI3, ULBP3 and MAP3K11 pI value is greater than 7. Hence, these all are basic in nature. $\mathrm{pI}$ value may be utilized for making buffer system when these proteins are to be purified in solution by isoelectric focusing method [6] [7] [8].

Table 2. Amino Acids (AA) Composition (in \%) of ERK Interacting Proteins Followed ExPASy server.

\begin{tabular}{|c|c|c|c|c|c|c|c|c|c|c|}
\hline $\mathrm{AA}$ & EPHB2 & MAPK1 & IL17RD & WDR83 & TESC & MAP3K4 & KSR2 & MAP3K6 & ULBP2 & ULBP1 \\
\hline Ala & $7.2 \%$ & $6.9 \%$ & $6.4 \%$ & $6.0 \%$ & $5.1 \%$ & $6.8 \%$ & $4.3 \%$ & $8.7 \%$ & $7.7 \%$ & $7.0 \%$ \\
\hline Arg & $5.5 \%$ & $5.3 \%$ & $4.7 \%$ & $6.7 \%$ & $7.0 \%$ & $6.2 \%$ & $6.0 \%$ & $7.9 \%$ & $4.1 \%$ & $3.7 \%$ \\
\hline Asn & $4.7 \%$ & $5.3 \%$ & $3.4 \%$ & $2.2 \%$ & $4.7 \%$ & $3.1 \%$ & $4.1 \%$ & $2.0 \%$ & $2.0 \%$ & $2.9 \%$ \\
\hline Asp & $5.2 \%$ & $6.7 \%$ & $5.3 \%$ & $7.6 \%$ & $7.0 \%$ & $5.7 \%$ & $3.8 \%$ & $4.0 \%$ & $4.9 \%$ & $4.5 \%$ \\
\hline Cys & $2.6 \%$ & $1.9 \%$ & $3.2 \%$ & $4.4 \%$ & $0.9 \%$ & $1.9 \%$ & $2.5 \%$ & $2.3 \%$ & $3.7 \%$ & $3.3 \%$ \\
\hline Gln & $4.5 \%$ & $3.3 \%$ & $5.0 \%$ & $3.2 \%$ & $3.7 \%$ & $4.5 \%$ & $4.8 \%$ & $6.0 \%$ & $4.1 \%$ & $4.5 \%$ \\
\hline Glu & $6.0 \%$ & $5.8 \%$ & $5.7 \%$ & $6.0 \%$ & $11.7 \%$ & $8.6 \%$ & $7.1 \%$ & $6.7 \%$ & $4.9 \%$ & $6.1 \%$ \\
\hline Gly & $6.9 \%$ & $4.7 \%$ & $6.9 \%$ & $10.2 \%$ & $5.6 \%$ & $5.5 \%$ & $4.6 \%$ & $7.1 \%$ & $6.5 \%$ & $5.3 \%$ \\
\hline His & $1.5 \%$ & $3.6 \%$ & $3.2 \%$ & $1.9 \%$ & $2.8 \%$ & $2.5 \%$ & $3.9 \%$ & $2.4 \%$ & $2.0 \%$ & $3.3 \%$ \\
\hline Ile & $6.4 \%$ & $7.8 \%$ & $3.5 \%$ & $1.6 \%$ & $6.5 \%$ & $5.1 \%$ & $5.1 \%$ & $3.3 \%$ & $4.9 \%$ & $3.7 \%$ \\
\hline Leu & $6.9 \%$ & $11.9 \%$ & $9.6 \%$ & $10.5 \%$ & $7.9 \%$ & $9.5 \%$ & $9.5 \%$ & $12.9 \%$ & $12.6 \%$ & $13.1 \%$ \\
\hline Lys & $3.9 \%$ & $6.4 \%$ & $5.8 \%$ & $4.4 \%$ & $4.7 \%$ & $6.4 \%$ & $6.8 \%$ & $2.4 \%$ & $5.7 \%$ & $7.0 \%$ \\
\hline Met & $3.5 \%$ & $2.5 \%$ & $1.8 \%$ & $1.0 \%$ & $5.1 \%$ & $2.7 \%$ & $1.9 \%$ & $1.6 \%$ & $3.7 \%$ & $3.3 \%$ \\
\hline Phe & $3.9 \%$ & $3.6 \%$ & $5.3 \%$ & $2.9 \%$ & $6.1 \%$ & $3.6 \%$ & $3.3 \%$ & $3.2 \%$ & $4.1 \%$ & $5.7 \%$ \\
\hline Pro & $4.8 \%$ & $5.6 \%$ & $6.0 \%$ & $4.1 \%$ & $3.3 \%$ & $6.2 \%$ & $9.7 \%$ & $7.9 \%$ & $6.1 \%$ & $6.1 \%$ \\
\hline Ser & $7.7 \%$ & $4.4 \%$ & $9.5 \%$ & $8.9 \%$ & $7.0 \%$ & $8.2 \%$ & $8.2 \%$ & $7.1 \%$ & $6.5 \%$ & $4.9 \%$ \\
\hline Thr & $6.4 \%$ & $3.9 \%$ & $4.3 \%$ & $5.1 \%$ & $4.2 \%$ & $4.5 \%$ & $6.0 \%$ & $4.8 \%$ & $7.3 \%$ & $6.1 \%$ \\
\hline $\operatorname{Trp}$ & $1.4 \%$ & $0.8 \%$ & $1.1 \%$ & $1.6 \%$ & $0.5 \%$ & $1.3 \%$ & $1.5 \%$ & $1.0 \%$ & $2.8 \%$ & $4.1 \%$ \\
\hline Tyr & $4.0 \%$ & $5.3 \%$ & $3.2 \%$ & $2.9 \%$ & $1.9 \%$ & $2.5 \%$ & $1.8 \%$ & $2.8 \%$ & $1.6 \%$ & $1.2 \%$ \\
\hline Val & $7.3 \%$ & $4.2 \%$ & $6.1 \%$ & $8.9 \%$ & $4.2 \%$ & $5.2 \%$ & $5.2 \%$ & $5.9 \%$ & $4.9 \%$ & $4.1 \%$ \\
\hline Pyl & $0.0 \%$ & $0.0 \%$ & $0.0 \%$ & $0.0 \%$ & $0.0 \%$ & $0.0 \%$ & $0.0 \%$ & $0.0 \%$ & $0.0 \%$ & $0.0 \%$ \\
\hline $\mathrm{Sec}$ & $0.0 \%$ & $0.0 \%$ & $0.0 \%$ & $0.0 \%$ & $0.0 \%$ & $0.0 \%$ & $0.0 \%$ & $0.0 \%$ & $0.0 \%$ & $0.0 \%$ \\
\hline
\end{tabular}


Table 3. Amino Acids (AA) Composition (in \%) of ERK Interactive Proteins Followed ExPASy server.

\begin{tabular}{|c|c|c|c|c|c|c|c|c|c|c|c|c|}
\hline $\mathrm{AA}$ & DUSP9 & MAP3K1 & ULBP3 & SLAMF7 & MAP3K11 & RHAMM & DUSP14 & DUSP6 & KIDINS220 & MAGI3 & PEA15 & WARS \\
\hline Ala & $8.3 \%$ & $8.4 \%$ & $7.8 \%$ & $3.9 \%$ & $8.4 \%$ & $5.9 \%$ & $7.6 \%$ & $4.5 \%$ & $6.1 \%$ & $4.9 \%$ & $3.8 \%$ & $7.2 \%$ \\
\hline Arg & $8.1 \%$ & $6.4 \%$ & $6.6 \%$ & $3.9 \%$ & $9.3 \%$ & $3.6 \%$ & $7.1 \%$ & $4.7 \%$ & $5.5 \%$ & $5.1 \%$ & $4.6 \%$ & $4.2 \%$ \\
\hline Asn & $3.4 \%$ & $3.8 \%$ & $1.6 \%$ & $5.1 \%$ & $1.8 \%$ & $5.2 \%$ & $3.5 \%$ & $6.3 \%$ & $4.9 \%$ & $4.5 \%$ & $3.1 \%$ & $3.6 \%$ \\
\hline Asp & $3.9 \%$ & $3.8 \%$ & $5.7 \%$ & $3.9 \%$ & $4.5 \%$ & $4.0 \%$ & $3.0 \%$ & $6.6 \%$ & $5.7 \%$ & $5.8 \%$ & $7.7 \%$ & $8.3 \%$ \\
\hline Cys & $2.1 \%$ & $2.4 \%$ & $2.5 \%$ & $2.7 \%$ & $1.3 \%$ & $1.1 \%$ & $2.0 \%$ & $2.4 \%$ & $1.6 \%$ & $1.1 \%$ & $0.8 \%$ & $1.3 \%$ \\
\hline Gln & $3.6 \%$ & $4.5 \%$ & $4.5 \%$ & $3.0 \%$ & $4.1 \%$ & $8.8 \%$ & $3.0 \%$ & $3.9 \%$ & $3.6 \%$ & $4.9 \%$ & $2.3 \%$ & $4.2 \%$ \\
\hline Glu & $7.3 \%$ & $6.9 \%$ & $5.3 \%$ & $6.9 \%$ & $7.4 \%$ & $15.2 \%$ & $3.5 \%$ & $6.3 \%$ & $6.5 \%$ & $7.6 \%$ & $12.3 \%$ & $5.1 \%$ \\
\hline Gly & $8.6 \%$ & $6.5 \%$ & $6.1 \%$ & $6.3 \%$ & $9.4 \%$ & $2.2 \%$ & $7.1 \%$ & $6.3 \%$ & $6.5 \%$ & $8.6 \%$ & $1.5 \%$ & $5.5 \%$ \\
\hline His & $1.6 \%$ & $2.5 \%$ & $2.5 \%$ & $2.1 \%$ & $1.4 \%$ & $1.5 \%$ & $4.0 \%$ & $1.0 \%$ & $2.3 \%$ & $2.5 \%$ & $1.5 \%$ & $2.3 \%$ \\
\hline Ile & $2.3 \%$ & $4.0 \%$ & $4.1 \%$ & $6.0 \%$ & $3.0 \%$ & $3.4 \%$ & $7.6 \%$ & $4.7 \%$ & $5.7 \%$ & $5.2 \%$ & $9.2 \%$ & $6.8 \%$ \\
\hline Leu & $14.8 \%$ & $9.0 \%$ & $12.3 \%$ & $10.7 \%$ & $9.6 \%$ & $13.8 \%$ & $8.1 \%$ & $12.6 \%$ & $11.0 \%$ & $7.2 \%$ & $11.5 \%$ & $7.2 \%$ \\
\hline Lys & $2.1 \%$ & $4.5 \%$ & $5.3 \%$ & $5.7 \%$ & $3.1 \%$ & $13.7 \%$ & $4.0 \%$ & $3.9 \%$ & $5.8 \%$ & $8.8 \%$ & $10.0 \%$ & $7.4 \%$ \\
\hline Met & $1.3 \%$ & $2.4 \%$ & $3.7 \%$ & $2.1 \%$ & $1.5 \%$ & $1.8 \%$ & $3.5 \%$ & $2.1 \%$ & $2.0 \%$ & $1.7 \%$ & $1.5 \%$ & $2.8 \%$ \\
\hline Phe & $3.9 \%$ & $2.6 \%$ & $5.3 \%$ & $2.7 \%$ & $2.8 \%$ & $2.2 \%$ & $3.5 \%$ & $5.0 \%$ & $2.9 \%$ & $2.4 \%$ & $2.3 \%$ & $7.0 \%$ \\
\hline Pro & $8.1 \%$ & $7.0 \%$ & $5.3 \%$ & $6.3 \%$ & $12.0 \%$ & $1.5 \%$ & $6.1 \%$ & $5.5 \%$ & $5.4 \%$ & $7.6 \%$ & $5.4 \%$ & $5.3 \%$ \\
\hline Ser & $10.2 \%$ & $12.0 \%$ & $7.0 \%$ & $8.4 \%$ & $8.0 \%$ & $7.0 \%$ & $7.1 \%$ & $11.3 \%$ & $9.7 \%$ & $8.2 \%$ & $8.5 \%$ & $7.2 \%$ \\
\hline Thr & $2.6 \%$ & $5.4 \%$ & $5.3 \%$ & $7.8 \%$ & $3.9 \%$ & $3.9 \%$ & $5.1 \%$ & $4.5 \%$ & $5.7 \%$ & $5.0 \%$ & $6.9 \%$ & $5.5 \%$ \\
\hline $\operatorname{Trp}$ & $0.8 \%$ & $0.9 \%$ & $3.7 \%$ & $1.8 \%$ & $2.5 \%$ & $0.3 \%$ & $2.0 \%$ & $0.8 \%$ & $1.2 \%$ & $0.7 \%$ & $0.8 \%$ & $0.6 \%$ \\
\hline Tyr & $2.6 \%$ & $1.6 \%$ & $2.0 \%$ & $3.6 \%$ & $1.2 \%$ & $1.4 \%$ & $4.0 \%$ & $2.6 \%$ & $2.5 \%$ & $1.8 \%$ & $3.1 \%$ & $3.2 \%$ \\
\hline Val & $4.4 \%$ & $5.6 \%$ & $3.3 \%$ & $7.5 \%$ & $4.7 \%$ & $3.4 \%$ & $8.1 \%$ & $5.0 \%$ & $5.5 \%$ & $6.4 \%$ & $3.1 \%$ & $5.1 \%$ \\
\hline Pyl & $0.0 \%$ & $0.0 \%$ & $0.0 \%$ & $0.0 \%$ & $0.0 \%$ & $0.0 \%$ & $0.0 \%$ & $0.0 \%$ & $0.0 \%$ & $0.0 \%$ & $0.0 \%$ & $0.0 \%$ \\
\hline $\mathrm{Sec}$ & $0.0 \%$ & $0.0 \%$ & $0.0 \%$ & $0.0 \%$ & $0.0 \%$ & $0.0 \%$ & $0.0 \%$ & $0.0 \%$ & $0.0 \%$ & $0.0 \%$ & $0.0 \%$ & $0.0 \%$ \\
\hline
\end{tabular}

\subsection{Extinction Coefficients (EC)}

The extinction coefficient of ERK interacting proteins at 280nm ranged from $11460 \mathrm{M}^{-1} \cdot \mathrm{cm}^{-1}$ to $188310 \mathrm{M}^{-1} \cdot \mathrm{cm}^{-1}$ in comparison to the concentration of Cys, Trp and Tyr (Table 4). Extinction coefficients of KIDINS220, MAP3K4, EPHB2, MAP3K11, MAP3K6, MAP3K, KSR2 were very higher indicating presence of high concentration of aromatic amino acid. Extinction coefficient of PEA15 was as lower $11460 \mathrm{M}^{-1} \cdot \mathrm{cm}^{-1}$ indicating that the presence of aromatic amino acids is low. The EC values are useful in the quantitative study of protein-protein and protein-ligand interactions in solution [6] [7] [8].

\subsection{The Instability Index (II)}

It gives estimation of protein stability in vitro. Protein having II value less than 40 the protein is measured as stable in laboratory condition whereas proteins II value higher than 40 is defined as unstable. ExPASy ProtParam result for II of ERK interacting proteins represent that only 4 proteins out of 22 is stable and remaining 17 are unstable [6] [7] [8]. 
Table 4. Physiochemical properties of Interacting Proteins of ERK.

\begin{tabular}{|c|c|c|c|c|c|c|c|c|c|c|}
\hline S.N. & Protein & $\begin{array}{c}\text { Number } \\
\text { of } \\
\text { amino } \\
\text { acids }\end{array}$ & $\begin{array}{c}\text { Molecular } \\
\text { weight }\end{array}$ & $\begin{array}{c}\text { Theoretical } \\
\text { pI }\end{array}$ & $\begin{array}{c}\text { Total number } \\
\text { of negatively } \\
\text { charged } \\
\text { residues } \\
\text { (Asp + Glu) }\end{array}$ & $\begin{array}{l}\text { Total } \\
\text { number of } \\
\text { positively } \\
\text { charged } \\
\text { residues } \\
\text { (Arg + Lys) }\end{array}$ & $\begin{array}{c}\text { Extinction } \\
\text { coefficients } \\
\mathrm{M}^{-1} \cdot \mathrm{cm}^{-1} \text {, at } \\
280\end{array}$ & $\begin{array}{c}\text { The } \\
\text { instability } \\
\text { index (II) }\end{array}$ & $\begin{array}{c}\text { Aliphatic } \\
\text { index }\end{array}$ & $\begin{array}{l}\text { Grand } \\
\text { average of } \\
\text { hydropa- } \\
\text { thicity } \\
\text { (GRAVY) }\end{array}$ \\
\hline 1 & EPHB2 & 987 & 110030.2 & 5.49 & 110 & 92 & 136735 & $\begin{array}{c}37.88 \\
\text { stable }\end{array}$ & 80.11 & -0.202 \\
\hline 2 & MAPK1 & 360 & 41389.7 & 6.50 & 45 & 42 & 45185 & $\begin{array}{l}39.71 \\
\text { stable }\end{array}$ & 95.94 & -0.287 \\
\hline 3 & IL17RD & 739 & 82410.5 & 6.78 & 81 & 78 & 81260 & $\begin{array}{c}56.54 \\
\text { Unstable }\end{array}$ & 75.21 & -0.346 \\
\hline 4 & WDR83 & 315 & 34342.6 & 5.36 & 43 & 35 & 41785 & $\begin{array}{c}42.56 \\
\text { Unstable }\end{array}$ & 78.86 & -0.305 \\
\hline 5 & TESC & 214 & 24749.8 & 4.84 & 40 & 25 & 11585 & $\begin{array}{c}49.58 \\
\text { Unstable }\end{array}$ & 73.83 & -0.568 \\
\hline 6 & MAP3K4 & 1608 & 181691.7 & 5.92 & 230 & 203 & 176975 & $\begin{array}{c}51.45 \\
\text { Unstable }\end{array}$ & 78.81 & -0.509 \\
\hline 7 & KSR2 & 950 & 107632.3 & 8.95 & 103 & 122 & 103830 & $\begin{array}{c}61.10 \\
\text { Unstable }\end{array}$ & 75.93 & -0.600 \\
\hline 8 & MAP3К6 & 1288 & 142596.0 & 6.70 & 133 & 138 & 127015 & $\begin{array}{c}58.54 \\
\text { Unstable }\end{array}$ & 88.79 & -0.254 \\
\hline 9 & ULBP2 & 246 & 27367.9 & 6.93 & 24 & 24 & 44960 & $\begin{array}{c}49.34 \\
\text { Unstable }\end{array}$ & 90.04 & 0.019 \\
\hline 10 & ULBP1 & 244 & 27996.5 & 7.07 & 26 & 26 & 59970 & $\begin{array}{c}40.06 \\
\text { Unstable }\end{array}$ & 84.39 & -0.162 \\
\hline 11 & DUSP14 & 198 & 42333.7 & 9.62 & 13 & 22 & 34170 & $\begin{array}{l}37.79 \\
\text { Stable }\end{array}$ & 92.07 & -0.012 \\
\hline 12 & DUSP6 & 381 & 22254.9 & 4.75 & 49 & 33 & 31900 & $\begin{array}{c}52.25 \\
\text { Unstable }\end{array}$ & 86.48 & 0.265 \\
\hline 13 & RHAMM & 725 & 84175.5 & 5.68 & 139 & 125 & 26400 & $\begin{array}{c}53.72 \\
\text { Unstable }\end{array}$ & 83.17 & -0.988 \\
\hline 14 & KIDINS220 & 1771 & 196542.1 & 6.19 & 217 & 199 & 188310 & $\begin{array}{c}44.54 \\
\text { Unstable }\end{array}$ & 87.17 & -0.369 \\
\hline 15 & MAGI3 & 1481 & 162948.8 & 8.26 & 199 & 205 & 96230 & $\begin{array}{c}48.35 \\
\text { Unstable }\end{array}$ & 71.65 & -0.746 \\
\hline 16 & PEA15 & 130 & 15068.1 & 4.93 & 26 & 19 & 11460 & $\begin{array}{c}64.55 \\
\text { Unstable }\end{array}$ & 93.77 & -0.625 \\
\hline 17 & WARS & 471 & 53178.5 & 5.83 & 63 & 55 & 39225 & $\begin{array}{c}40.34 \\
\text { Unstable }\end{array}$ & 76.65 & -0.345 \\
\hline 18 & DUSP9 & 384 & 41934.6 & 5.96 & 43 & 39 & 31900 & $\begin{array}{c}67.23 \\
\text { Unstable }\end{array}$ & 88.20 & -0.245 \\
\hline 19 & MAP3K1 & 1512 & 164469.9 & 7.93 & 161 & 165 & 115010 & $\begin{array}{c}63.95 \\
\text { Unstable }\end{array}$ & 75.07 & -0.425 \\
\hline 20 & ULBP3 & 244 & 27949.3 & 8.20 & 27 & 29 & 57325 & $\begin{array}{c}46.78 \\
\text { Unstable }\end{array}$ & 81.23 & -0.236 \\
\hline 21 & SLAMF7 & 335 & 37420.9 & 6.02 & 36 & 32 & 51380 & $\begin{array}{l}37.55 \\
\text { Stable }\end{array}$ & 90.72 & -0.188 \\
\hline 22 & MAP3K11 & 847 & 92687.9 & 8.40 & 101 & 105 & 131025 & $\begin{array}{c}70.83 \\
\text { Unstable }\end{array}$ & 70.89 & -0.582 \\
\hline
\end{tabular}




\subsection{Aliphatic Index}

Aliphatic index of a protein is considered as the relative volume captured by aliphatic side chains (alanine, valine, isoleucine, and leucine). It is considered as a positive factor for the increase of thermal stability of annular proteins. Aliphatic index of ERK interactive proteins limit from 62.34 to 107.02. Both MAP3K11 and MAGI3 have lower thermal stability representing their more flexible structure as compared to other proteins (Table 4). The high aliphatic index of other proteins indicates their stability in high range of temperature [6] [7] [8].

\subsection{A GRAVY (Grand Average of Hydropathy)}

GRAVY is understood as the total hydropathy values of all the amino acids of a protein, divided by the number of amino acids residues in the sequence. ExPASy Protparam server for GRAVY indicated that ERK interactive proteins are hydrophilic in nature except ULBP2. Lowest GRAVY value of DUSP14 suggests its better communication with water [6] [7] [8].

\subsection{Functional Characterization}

The SOSUI server data expressed ERK interacting proteins namely EPHB2 (2 trans membrane), IL17RD (2 trans membrane), ULBP2 (2 trans membrane), ULBP1 (1 trans membrane), KIDINS220 (4 trans membrane) and SLAMF7 (2 trans membrane) are membrane protein. However, MAPK1, WDR83, TESC, MAP3K4, KSR2, MAP3K6, DUSP6, DUSP14, MAGI3, PEA15, WARS, DUSP9, MAP3K1, ULBP3, MAP3K11 and DUSP4 are classified as a soluble protein (Table 5).

\section{Discussion}

There are several genes responsible for the long term synaptic plasticity. ERK is one of these gene involved in long term learning and memory. During this process, a cascade of regulatory immediate early genes is recruited to particular gene including ERK [11] [12]. These genes are required for long-term synaptic plasticity and long-term memory formation. For gene expression, chromatin remodeling is crucial event. Studies from several researchers have established that chromatin remodeling is involved in learning and memory, long-term neuronal responses, drug addiction, stress, epilepsy and depression [13] [14]. For performing any functions ERK interacts with a host of proteins. Hence the ERK interacting proteins are crucial and involved in almost all brain related disorders and diseases.

The chromatin remodeling is done by modification at the level of histones like acetylation and methylation. histone acetylation is a reversible modification of lysine residues within the amino-terminal domains histone. It is done by histone acetyltransferase (HAT), which transfers acetyl groups from acetyl-coenzyme A to the lysine residue. In the other hand, histone deacetylase (HDAC) acts in the 
Table 5. Functional characterization of ERK interactive proteins.

\begin{tabular}{|c|c|c|c|c|c|c|c|c|}
\hline No. & $\begin{array}{c}\text { Interactive } \\
\text { Proteins }\end{array}$ & $\begin{array}{c}\mathrm{N} \\
\text { terminal }\end{array}$ & Trans-membrane region & C terminal & Type & Length & & Characters \\
\hline 1 & \multirow[b]{2}{*}{ EPHB2 } & 6 & LGAALLLLPLLAAVEETLMDSTT & 28 & SECONDARY & 23 & & MEMBRANE \\
\hline \multirow[t]{2}{*}{2} & & 542 & PLIIGSSAAGLVFLIAVVVIAIV & 564 & PRIMERY & 23 & & $\begin{array}{c}\text { PROTEIN } \\
2 \text { Transmembrane } \\
\text { helices. }\end{array}$ \\
\hline & MAPK1 & - & - & - & - & - & & SOLUBLE PROTEIN \\
\hline 1 & \multirow[b]{2}{*}{ IL17RD } & 1 & MAPWLQLCSVFFTVNACLNGSQL & 23 & SECONDARY & 23 & & MEMBRANE \\
\hline \multirow[t]{6}{*}{2} & & 298 & IRAVAITVPLVVISAFATLFTVM & 320 & PRIMARY & 23 & & $\begin{array}{l}2 \text { Transmembrane } \\
\text { helices. }\end{array}$ \\
\hline & WDR83 & - & - & - & - & - & & SOLUBLE PROTEIN \\
\hline & TESC & - & - & - & - & - & & SOLUBLE PROTEIN \\
\hline & MAP3K4 & - & - & - & - & - & & SOLUBLE PROTEIN \\
\hline & KSR2 & - & - & - & - & - & & SOLUBLE PROTEIN \\
\hline & MAP3K6 & - & - & - & - & - & & SOLUBLE PROTEIN \\
\hline \multirow{5}{*}{2} & ULBP? & 2 & AAAAATKILLCLPLLLLLSGWSR & & 24 & PRIMARY & 23 & $\begin{array}{c}\text { MEMBRANE } \\
\text { PROTEIN }\end{array}$ \\
\hline & ULBP2 & 225 & TATTLILCCLLIILPCFILPGI & & 246 & PRIMARY & 22 & $\begin{array}{c}2 \text { Transmembrane } \\
\text { helices. }\end{array}$ \\
\hline & ULBP1 & 1 & MAAAASPAFLLCLPLLHLLSGWS & & 23 & PRIMARY & 23 & $\begin{array}{c}\text { MEMBRANE } \\
\text { PROTEIN } \\
1 \text { Transmembrane } \\
\text { helices }\end{array}$ \\
\hline & DUSP14 & - & - & & - & - & - & SOLUBLE PROTEIN \\
\hline & DUSP6 & - & - & & - & - & - & SOLUBLE PROTEIN \\
\hline 1 & \multirow{4}{*}{ KIDINS220 } & 496 & QFSWLIVFLTLLLCGGLGLLFAF & & 518 & PRIMARY & 23 & $\begin{array}{c}\text { MEMBRANE } \\
\text { PROTEIN }\end{array}$ \\
\hline 2 & & 525 & GIAVSLSFLALLYIFFIVIYFGG & & 547 & PRIMARY & 23 & $\begin{array}{c}4 \text { Transmembrane } \\
\text { helices }\end{array}$ \\
\hline 3 & & 657 & LPSFVIFLFIIGCIISGITLLAI & & 679 & PRIMARY & 23 & \\
\hline \multirow[t]{7}{*}{4} & & 687 & LTVNAVLISIASVVGLAFVLNCR & & 709 & PRIMARY & 23 & \\
\hline & MAGI3 & - & - & & - & - & - & SOLUBLE PROTEIN \\
\hline & PEA15 & - & - & & - & - & - & SOLUBLE PROTEIN \\
\hline & WARS & - & - & & - & - & - & SOLUBLE PROTEIN \\
\hline & DUSP9 & - & - & & - & - & - & $\begin{array}{l}\text { SOLUBLE } \\
\text { PROTEIN }\end{array}$ \\
\hline & MAP3K1 & - & - & & - & - & - & $\begin{array}{l}\text { SOLUBLE } \\
\text { PROTEIN }\end{array}$ \\
\hline & ULBP3 & - & - & & - & - & - & SOLUBLE PROTEIN \\
\hline 1 & \multirow{2}{*}{ SLAMF7 } & 1 & MAGSPTCLTLIYILWQLTGSAAS & & 23 & $\begin{array}{l}\text { SECONDAR } \\
\mathrm{Y}\end{array}$ & 23 & $\begin{array}{l}\text { MEMBRANE } \\
\text { PROTEIN }\end{array}$ \\
\hline 2 & & 226 & MVLLCLLLVPLLLSLFVLGLFLW & & 248 & PRIMARY & 23 & $\begin{array}{c}2 \text { Transmembrane } \\
\text { helices }\end{array}$ \\
\hline & MAP3K11 & - & - & & - & - & - & SOLUBLE PROTEIN \\
\hline & DUSP14 & - & - & & - & - & - & $\begin{array}{l}\text { SOLUBLE } \\
\text { PROTEIN }\end{array}$ \\
\hline
\end{tabular}


reverse manner and removes the acetyl groups from lysine residues. Actually, acetylation neutralizes the basic histone tails by weakening of the histone-DNA interaction and promoting the accessibility of transcription factors. The present study revealed that all ERK interacting proteins are rich in lysine residues i.e. basic in nature. Further ERK interacting proteins are hydrophobic in nature which favors gene expression at transcriptional level. So these proteins may be involved in histone modification needed for chromatin dynamics and gene transcription. Furthermore, there are several kinases which are decisively involved in histone phosphorylation affecting long-term memory formation nemely ERK1/2, p38 MAP kinase, and ribosomal 6 kinase (RSK) [14]. The aforementioned findings favor our result as ERK interacted with various proteins as shown in this paper.

In addition, calsyntenins (Cst) comprise a family of trans-membrane proteins with the unique potential to link extracellular proteolytic activity with intracellular calcium signaling. It is molecular class of calcium binding protein, which can bind calcium ion and being involved in cell signaling and cell-cell communication. It contains three members, calsyntenin-1, calsyntenin- 2 and calsyntenin-3, which are postsynaptic membrane proteins and predominantly expressed in brain neurons. Each of the three calsyntenins exhibits a distinct neuronal mRNA expression pattern. The calsyntenin-1 is located in the postsynaptic membrane of CNS and a proteolytically processed protein of the postsynaptic membrane contains calcium-binding cytoplasmic domain. Calsyntenin-1 is a dynamic modulator of postsynaptic calcium by extracellular proteolysis. It may modulate $\mathrm{Ca}^{2+}$ transients locally either beneath the postsynaptic membrane or around intracellular $\mathrm{Ca}^{2+}$ stores, such as LTP and LTD. Recent studies showed that impairment of the coordinated metabolic regulation of APP, calsyntenins and the consequent loss of the reciprocal regulation by APP and calsyntenins in the gene transactivation in $\mathrm{AD}$ [15]. Almost all ERK interacting proteins are trans-membrane in nature. Thus ERK interacting proteins may be involved in metabolic regulation of APP and calsyntenins mediated pathway of AD.

Food provides energy and building blocks to the body. Its ability to prevent and protect against diseases is started to be recognized. Especially research over the past one decade has provided exciting evidence suggests the influence of dietary factors on brain functions. For example, omega- 3 fatty acids rich diet is useful for cognitive processes in humans [16] and up-regulating genes that are significant for maintaining synaptic function and plasticity in rodents [17]. Further, high unsaturated fat diets are responsible for reducing molecular substrates that support cognitive processing and increases the risk of neurological dysfunctions in both humans and animals [18] [19]. The important effects of food on brain functions are cited still further research is necessary to determine underlying mechanisms of action and for therapeutic applications in humans. In this study, we found that most of the ERK interacting are rich in leucine, suggesting that their roles as transcription factor. The leucine zipper motif contains leucine residues spaced 7 amino acids apart repeating at least 3 times $(\mathrm{L} \times \times \times \times \times \times L \times \times \times \times \times \times L)$ 
[20]. In the process of dimerization, parallel leucine zipper motifs interact through a coiled coil hydrophobic interface that juxtaposes 2 adjacent basic regions [21]. Further, once bound to DNA, these adjacent basic regions undergo key changes in conformation [22]. As almost ERK interacting proteins contain leucine so it may be assumed that these are involved in ERK mediated functions including learning and memory. In addition, leucine rich food could be good for cognition and healthy brain functions. Thus such findings regarding ERK interacting proteins are instrumental to understand learning and memory via ERK. Moreover, it may be helpful to target these interacting proteins during learning and memory deficit and neurodegenerative diseases.

\section{Acknowledgements}

VP acknowledges Science and Engineering Research Board (SERB), Government of India for providing financial support (Registration No.SERB/LS-485/2013). KK acknowledges SERB for Junior Research Fellowship.

\section{References}

[1] Ebisuya, M., Kondoh, K. and Nishida, E. (2005) The Duration, Magnitude and Compartmentalization of ERK MAP Kinase Activity: Mechanisms for Providing Signaling Specificity. Journal of Cell Science, 118, 2997-3002. https://doi.org/10.1242/jcs.02505

[2] Vidhya, V.G., Upgade, A., Bhaskar, A. and Deb, D. (2012) In Silico Characterization of Bovine (Bostaurus) Antiapoptotic Proteins. Journal of Proteins and Proteomics, 3, 187-196.

[3] Kuhn, M., Mering, V.C., Campillos, M., Jensen, J.L. and Bork, P. (2015) STITCH: Interaction Networks of Chemicals and Proteins. Nucleic Acids Research, 36, D684-D688. https://doi.org/10.1093/nar/gkm795

[4] Szklarczyk, D., Franceschini, A., Wyder, S., Forslund, K., Heller, D., Huerta. C.J., Simonovic, M., Alexander, R., Santos, A., Tsafou, P.K., Kuhn, M., Bork, P., Jensen, J.L. and Mering, V.C. (2015) STRING v10: Protein-Protein Interaction Networks, Integrated over the Tree of Life. Nucleic Acids Research, 43, D447-D452. https://doi.org/10.1093/nar/gku1003

[5] Bidkar, A.P., Thakur, K.K., Bolshette, NB., Dutta, J. and Gogoi, R. (2014) In-Silico Structural and Functional Analysis of Hypothetical Proteins of Leptospira Interrogans. Biochemistry \& Pharmacology, 3, 3. https://doi.org/10.4172/2167-0501.1000136

[6] Jabalia, N., Bansal, H., Mishra, P.C. and Chaudhary, N. (2015) In-Silico Comparative Analysis of Papain Family Cysteine Protease Using Computational Tools and Servers. International Journal of Basic and Applied Biology, 2, 310-314.

[7] Mahalakshmi, K. (2015) Insilico Analysis of Proteins of Curcuma aromatica Salisb. International Journal of Pharm Tech Research, 8, 51-56.

[8] Vishwanath, K.V., Pattabhiramaiah, M. and Keerthi, R. (2016) Bio Computational Analysis of Protein Sequence of Sickle Cell Anemia. International Journal of Engineering Research and General Science, 1, 63-73.

[9] Verma, K.N., Verma, V., Deshwal R.K. and Yadav, N. (2014) Structural Insight into Molecular Model of Hypothetical Protein from Trichomonas vaginalis. A Compu- 
tational Approach. Biosciences, 76, 28414-28421.

[10] Hirokawa, T (1998) SOSUI: Classification and Secondary Structure Prediction System for Membrane Proteins. Bioinformatics, 14, 378-379.

https://doi.org/10.1093/bioinformatics/14.4.378

[11] Goelet, P., Castellucci, V.F., Schacher, S. and Kandel E.R. (1986) The Long and the Short of Long-Term Memory-A Molecular Framework. Nature, 322, 419-422. https://doi.org/10.1038/322419a0

[12] Taubenfeld, S.M., Milekic, M.H., Monti, B. and Alberini, C.M. (2001) The Consolidation of New but Not Reactivated Memory Requires Hippocampal C/EBP $\beta$. Nature Neuroscience, 4, 813-818. https://doi.org/10.1038/90520

[13] McClung, C.A. and Nestler, E.J. (2008) Neuroplasticity Mediated by Altered Gene Expression. Neuropsychopharmacology, 33, 3-17.

https://doi.org/10.1038/sj.npp.1301544

[14] Taniura, H., Sng, J.C. and Yoneda, Y. (2008) Histone Modifications in the Brain. Neurochemistry International, 51, 85-91. https://doi.org/10.1016/j.neuint.2007.04.018

[15] Cheng, X.R., Zhou, W.X. and Zhang, Y.X. (2006) The Family of Calsyntenins: Learning and Memory Related Genes. Progress in Physiology, 37, 205-210.

[16] McCann, J.C. and Ames, B.N. (2005) Is Docosahexaenoic Acid, an N-3 Long-Chain Polyunsaturated Fatty Acid, Required for Development of Normal Brain Function? An Overview of Evidence from Cognitive and Behavioral Tests in Humans and Animals. The American Journal of Clinical Nutrition, 82, 281-295. https://doi.org/10.1093/ajcn/82.2.281

[17] Wu, A., Ying, Z. and Gomez-Pinilla, F. (2007) Omega-3 Fatty Acids Supplementation Restores Mechanisms That Maintain Brain Homeostasis in Traumatic Brain Injury. Journal of Neurotrauma, 24, 587-1595. https://doi.org/10.1089/neu.2007.0313

[18] Greenwood, C.E. and Winocur, G. (2005) High-Fat Diets, Insulin Resistance and Declining Cognitive Function. Neurobiology of Aging, 26, 42-45. https://doi.org/10.1016/j.neurobiolaging.2005.08.017

[19] Molteni, R., Barnard, J.R., Ying, Z., Roberts, C.K. and Gomez-Pinilla, F. (2002) A High-Fat, Refined Sugar Diet Reduces Hippocampal Brain-Derived Neurotrophic Factor, Neuronal Plasticity and Learning. Neuroscience, 112, 803-814. https://doi.org/10.1016/S0306-4522(02)00123-9

[20] Landschulz, W.H., Johnson, P.F. and McKnight, S.L. (1988) The Leucine Zipper: A Hypothetical Structure Common to a New Class of DNA Binding Proteins. Science, 240, 1759-1764. https://doi.org/10.1126/science.3289117

[21] Ellenberger, T.E., Brandl, C.J., Struhl, K. and Harrison, S.C. (1992) The GCN4 Basic Region Leucine Zipper Binds DNA as a Dimer of Uninterrupted $\alpha$ Helices: Crystal Structure of the Protein-DNA Complex. Cell, 71, 1223-1237. https://doi.org/10.1016/S0092-8674(05)80070-4

[22] O’Neil, K.T. and DeGrado, W.F. (1990) A Thermodynamic Scale for the Helix-Forming Tendencies of the Commonly Occurring Amino Acids. Science, 250, 646-651. https://doi.org/10.1126/science.2237415 